\title{
Beneficial effect of a weight-stable, low-fat/low-saturated fat/low-glycaemic index diet to reduce liver fat in older subjects
}

\author{
Kristina M. Utzschneider ${ }^{1,2 *}$, Jennifer L. Bayer-Carter ${ }^{3,4}$, Matthew D. Arbuckle ${ }^{3}$, Jaime M. Tidwell ${ }^{3}$, \\ Todd L. Richards ${ }^{5}$ and Suzanne Craft ${ }^{3,4}$ \\ ${ }^{1}$ Division of Metabolism, Endocrinology and Nutrition, Department of Medicine, VA Puget Sound Health Care System, \\ 1660 South Columbian Way, Seattle, WA 98108, USA \\ ${ }^{2}$ Division of Metabolism, Endocrinology and Nutrition, Department of Medicine, University of Washington, Seattle, \\ WA 98195, USA \\ ${ }^{3}$ Geriatric Research Education and Clinical Center (GRECC), VA Puget Sound Health Care System, Seattle, WA 98108, USA \\ ${ }^{4}$ Department of Psychiatry and Behavioral Sciences, University of Washington, Seattle, WA 98195, USA \\ ${ }^{5}$ Department of Radiology, University of Washington, Seattle, WA 98195, USA
}

(Submitted 30 January 2012 - Final revision received 10 May 2012 - Accepted 31 May 2012 - First published online 31 July 2012)

\begin{abstract}
Non-alcoholic fatty liver disease is associated with insulin resistance and dyslipidaemia and can progress to steatohepatitis and cirrhosis. We sought to determine whether dietary fat and saturated fat content alter liver fat in the absence of weight change in an older population. Liver fat was quantified by magnetic resonance spectroscopy before and after 4 weeks on an isoenergetic low-fat/low-saturated fat/low-glycaemic index (LGI) (LSAT: $23 \%$ fat/7\% saturated fat/GI < 55) or a high-fat/high-saturated fat/high-GI $($ HSAT: $43 \%$ fat $/ 24 \%$ saturated fat/GI > 70) diet in older subjects. In the present study, twenty subjects (seven males/thirteen females; age 69.3 (sEm 1.6) years, BMI $26.9($ SEM $0 \cdot 8) \mathrm{kg} / \mathrm{m}^{2}$ ) were randomised to the LSAT diet and fifteen subjects (six males/nine females; age 68.6 (SEM $1 \cdot 8$ ) years, BMI $28.1(\mathrm{SEM} 0.9) \mathrm{kg} / \mathrm{m}^{2}$ ) to the HSAT diet. Weight remained stable. Liver fat decreased significantly on the LSAT diet (median $2 \cdot 2$ (interquartile range (IQR) 3.1 ) to 1.7 (IQR 1.8$) \%, P=0.002$ ) but did not change on the HSAT diet (median 1.2 (IQR 4.1 ) to 1.6 (IQR 3.9 ) \%). The LSAT diet lowered fasting glucose and total cholesterol, HDL-cholesterol and LDL-cholesterol and raised TAG $(P<0 \cdot 05)$, while the HSAT diet had no effect on glucose or HDL-cholesterol but increased total cholesterol and LDL-cholesterol $(P<0 \cdot 05)$. Fasting insulin and homeostasis model of insulin resistance did not change significantly on either diet, but the Matsuda index of insulin sensitivity improved on the LSAT diet $(P<0 \cdot 05)$. Assignment to the LSAT $v$. HSAT diet was a predictor of changes in lipid parameters but not liver fat. We conclude that diet composition may be an important factor in the accumulation of liver fat, with a low-fat/low-saturated fat/LGI diet being beneficial.
\end{abstract}

Key words: Non-alcoholic fatty liver: Dietary fat: Glycaemic index: Saturated fat

Non-alcoholic fatty liver disease (NAFLD) is an increasingly common condition characterised by hepatic TAG accumulation in subjects who do not drink excess alcohol. The prevalence of NAFLD in an urban population with an average age of 46 years has been shown to be $33 \%{ }^{(1)}$, but no study has specifically examined the prevalence of NAFLD in the older population. This population is of interest as age appears to be an important factor in the progression from NAFLD to non-alcoholic steatohepatitis (NASH). In a recent study of patients diagnosed with NAFLD by liver biopsy, subjects $<50$ years old were more likely to have simple steatosis, while those aged 50-60 years were more likely to have $\mathrm{NASH}$, and those aged $>60$ years were more likely to have cirrhosis $^{(2)}$.

The role that the diet plays in the pathogenesis and treatment of NAFLD is incompletely understood. Dietary factors may contribute to liver fat accumulation by multiple pathways. First, the amount of dietary fat may directly affect liver fat content, with high-fat diets being potentially deleterious. In subjects with NAFLD fed a $3 \mathrm{~d}$ isoenergetic diet containing $30 \%$ energy from fat, $15 \%$ of the TAG in the liver was derived directly from dietary fat ${ }^{(3)}$. Dietary composition may also

Abbreviations: AD, Alzheimer's disease; DNL, de novo lipogenesis; GI, glycaemic index; HGI, high glycaemic index; HOMA-IR, homeostasis model of insulin resistance; HSAT, high-fat/high-saturated fat/high-glycaemic index diet; IAF, intra-abdominal fat; LGI, low glycaemic index; LSAT, low-fat/low-saturated fat/low-glycaemic index diet; MCI, mild cognitive impairment; MRS, magnetic resonance spectroscopy; NAFLD, non-alcoholic fatty liver disease; NASH, non-alcoholic steatohepatitis; SQF, subcutaneous fat.

*Corresponding author: K. M. Utzschneider, fax +1 206764 2164, email kutzschn@u.washington.edu 
contribute to liver fat accumulation by modulating insulin sensitivity. Diets high in saturated fat ${ }^{(4-6)}$ and simple carbohydrates $^{(7,8)}$ have been shown to induce insulin resistance. Adipose tissue insulin resistance is associated with increased $\mathrm{NEFA}^{(9)}$, and hyperinsulinaemia stimulates hepatic de novo lipogenesis $(\mathrm{DNL})^{(10)}$. Foods with a higher glycaemic index (GI), an index which reflects the degree to which food increases blood glucose ${ }^{(11)}$, may be particularly bad as they have greater effects to increase postprandial TAG and $\operatorname{NEFA}^{(12,13)}$

In epidemiological studies, both the total amount of fat and the amount of saturated fat in the diet have been correlated with liver TAG content ${ }^{(14)}$ and the presence of $\mathrm{NASH}^{(15)}$. Increased intake of simple carbohydrates ${ }^{(16)}$, soft drinks ${ }^{(17)}$ and a diet with a high GI (HGI) ${ }^{(18)}$ has also been associated with increased liver fat. These studies are limited by their cross-sectional nature, use of diet recall or food diaries to estimate dietary composition and inability to account for excess energy intake.

Energy balance is a major factor in liver fat accumulation. Overfeeding studies have clearly shown that increased intake of fat ${ }^{(19,20)}$, glucose $e^{(21)}$ or fructose ${ }^{(20,21)}$ can increase liver fat content in young, healthy, lean subjects. Conversely, weight loss has been shown to decrease liver fat content ${ }^{(22-25)}$ and improve liver histology ${ }^{(23,24)}$. In obese older adults, weight loss resulted in significant decreases in liver fat of $45 \%^{(26)}$. Studies examining the effect of diet composition on liver fat in the absence of weight gain or loss are lacking. To date, only two studies utilising weight-stable designs have investigated the impact of dietary fat and saturated fat content on liver fat. The first study was performed in pre-menopausal obese women $^{(27)}$ and the second one in middle-aged overweight men ${ }^{(28)}$. Both utilised a cross-over design and found that a low-fat diet decreased liver fat compared with a high-fat diet.

We sought to determine the effect of dietary fat content on liver fat accumulation in an older population. We hypothesised that a low-fat/low-saturated fat diet with a low GI (LGI) (LSAT) would decrease liver fat and improve insulin sensitivity and that a high-fat/high-saturated fat diet with a HGI (HSAT) would increase liver fat and decrease insulin sensitivity.

\section{Subjects and methods}

\section{Study design}

The present study was conducted as part of a larger study titled 'Macronutrient Effects on Alzheimer's Disease' (NIA R37 AG 10880) which sought to determine the impact of diet-induced insulin resistance on memory and cognitive function $^{(29)}$. It was a randomised, double-blind, parallel design diet intervention study comparing the effects of a 4 -week weightstable LSAT $v$. HSAT diet.

\section{Subjects}

Subjects were recruited from the Veterans Affairs Puget Sound Health Care System Memory Wellness Program, by referral from other Alzheimer's disease (AD) research centres and using targeted recruiting in the Greater Seattle area. Subjects with normal memory and cognition and subjects with mild cognitive impairment (MCI) or early AD were studied. Exclusion criteria included: diabetes or HbA1c $>6.4 \%$; liver enzymes $>1.5$ times normal; LDL-cholesterol $>4.92 \mathrm{~mm}$, TAG $>3.83 \mathrm{~mm}$, total cholesterol $>6.73 \mathrm{~mm}$ or LDL:HDL ratio $>3.0$ or the use of lipid-lowering medications; significant neurological disease other than $\mathrm{AD}$; significant medical disease or organ failure; current use of glucocorticoids, antipsychotics, anticonvulsants, anxiolytics, anti-coagulants, sedatives or cognitionenhancing medications (except cholinesterase inhibitors or Namenda $\left.{ }^{\circledR}\right)$; or relevant food allergies. Of the forty-nine subjects who completed the parent study, thirty-five eligible subjects participated in the magnetic resonance spectroscopy (MRS) sub-study and completed both pre- and post-diet scans. All subjects gave written informed consent. The present study was conducted according to the guidelines laid down in the Declaration of Helsinki and all procedures involving human subjects/patients were approved by the Institutional Review Boards of the Veterans Affairs Puget Sound Health Care System and the University of Washington.

\section{Dietary intervention}

Subjects were randomly assigned to receive either a LSAT ( $n$ 20: controls $n$ 9; MCI/AD $n$ 11) or a HSAT diet ( $n$ 15: controls $n 5$; MCI/AD $n$ 10). The diets were designed to contain the following macronutrient compositions: LSAT, 25\% energy from fat $/ 7 \%$ saturated fat $/ 15-20 \%$ protein $/ 55-60 \%$ carbohydrates/daily average GI index < 55 ; HSAT, $45 \%$ fat $/ 25 \%$ saturated fat $/ 15-20 \%$ protein $/ 35-40 \%$ carbohydrates/daily average GI index $>70$. Menus were designed by a research nutritionist and analysed by ProNutra (VioCare, Inc.). The daily average GI was calculated as described previously ${ }^{(11)}$ For the LSAT diet, the majority of the menu included low-fat and non-fat dairy products, lean meats and minimally processed grains to meet the saturated fat and GI goals. The HSAT diet emphasised butter, cheeses, fatty meats and cream to meet the saturated fat goals and refined carbohydrates to meet the GI goals. Treats and 'confusing foods' (such as bacon and Doritos ${ }^{\circledR}$ chips on the LSAT diet and watermelon and cottage cheese on the HSAT diet) were also included so people were less able to guess which diet they were on. A sample $1 \mathrm{~d}$ menu is provided in Table 1. Due to the lack of fructose data in the USDA and Food and Nutrient Database for Dietary Studies databases used by ProNutra (at most, 9.5\% of foods with fructose content), an accurate assessment of fructose content in the diets was not possible.

At baseline, subjects completed a physical activity questionnaire and a $3 \mathrm{~d}$ food record (two weekdays and one weekend day) to estimate the composition of their usual diet. Fructose values were estimated from the USDA Standard Reference 18 and Food and Nutrient Database for Dietary Studies 1.0 $0^{(30)}$. Energy needs were calculated using the average of the Mifflin-St Jeor ${ }^{(31)}$ and Harris-Benedict ${ }^{(32)}$ equations, adjusted for physical activity, and rounded up to the nearest $837 \mathrm{~kJ}$ (200 kcal). All food was prepared in a commercial metabolic 
Table 1. Sample menu for $1 \mathrm{~d}$ on the intervention diets

\begin{tabular}{|c|c|c|}
\hline & LSAT diet & HSAT diet \\
\hline Breakfast & $\begin{array}{l}\text { Steel cut oatmeal, cooked in } 2 \% \text { milk topped } \\
\text { with raspberries, blueberries, brown sugar, } \\
n-3 \text { margarine and pecans }\end{array}$ & $\begin{array}{l}\text { Cream of wheat, cooked in water and half and half } \\
\text { and topped with dates, syrup and butter }\end{array}$ \\
\hline Morning snack & High-protein granola bar & Cherry oat bar \\
\hline \multirow[t]{2}{*}{ Lunch } & $\begin{array}{l}\text { Grilled ham and swiss sandwich: low-fat ham and } \\
\text { light-swiss cheese on seedy pumpernickel } \\
\text { bread grilled in cooking spray }\end{array}$ & $\begin{array}{l}\text { Grilled beef and cheddar sandwich: roast beef and } \\
\text { cheddar cheese on white bread grilled in butter }\end{array}$ \\
\hline & $\begin{array}{l}\text { Minestrone soup } \\
\text { Peanut M\&Ms }\end{array}$ & Rice krispie treat \\
\hline \multirow[t]{2}{*}{ Afternoon snack } & $1 \%$ fat cottage cheese & Saltine crackers \\
\hline & Peaches, canned in fruit juice & Whole milk mozzarella cheese \\
\hline Dinner & $\begin{array}{l}\text { Asian chicken noodle bowl: chicken breast and } \\
\text { broccoli, pea pods, onions and carrots over } \\
\text { spaghetti with a soy ginger sauce }\end{array}$ & $\begin{array}{l}\text { Turkey thigh with gravy } \\
\text { Green beans with butter } \\
\text { Instant mashed potatoes reconstituted with } \\
\text { half and half and butter } \\
\text { Cranberry sauce }\end{array}$ \\
\hline Bedtime snack & $\begin{array}{l}\text { Lightly sweetened strawberries with low-fat pound } \\
\text { cake and low-fat whipped topping }\end{array}$ & Chocolate ice cream with whipped cream \\
\hline
\end{tabular}

LSAT, low fat/low saturated fat/low glycaemic index diet; HSAT, high fat/high saturated fat/high glycaemic index diet.

kitchen and delivered to participants' homes twice weekly for 4 weeks. Each subject was allowed one 'free' meal per week and a daily beverage (approximately $418 \mathrm{~kJ}$ or $100 \mathrm{kcal}$ ) from a limited list that included alcohol. To determine compliance, subjects recorded all food consumed each day and received a weekly phone call. All participants were compliant with macronutrient targets, and the number of non-compliant incidents was comparable between the groups (average range 1.23-1.8 incidents/week). Subjects were instructed to maintain their usual level of physical activity. Study procedures were performed at baseline and during the fourth week on the diet.

\section{Oral glucose tolerance test}

Glucose tolerance was assessed by a standard $75 \mathrm{~g}$ oral glucose load given after an overnight fast $(12 \mathrm{~h})$. Samples were collected at times $0,15,60$ and 120 min post-glucose ingestion.

\section{Quantification of liver fat}

MRS was used to quantify hepatic TAG using a Philips Achieva (3 Tesla, version 2.5.3.0) whole body scanner. Press sequences (repetition time (TR) $2000 \mathrm{~ms}$ and five echo times (TE) - TE $30,45,65,100$ and $200 \mathrm{~ms}$ ) with breath-holds for ten averages, a spectral width of $2000 \mathrm{~Hz}$ and a resolution of 2048 points being used. Sagittal, coronal and axial images were acquired through the liver and an $8 \mathrm{~cm}^{3}$ spectroscopic volume of interest was positioned in the right lobe of the liver to avoid major vessels or bile ducts. Lipid was measured based on the resonances for protons of water and methylene groups in lipids and then converted into absolute concentrations, expressed as percentage fat by weight, using methods similar to those validated by both Longo et al. ${ }^{(33)}$ and Szczepaniak et al. ${ }^{(34)}$. For each subject, three spectra were obtained at each of the first three echo times and the results averaged. MRS scans were interpreted by a single radiologist who was blinded to the diet intervention. The inter- and intra-scan CV for liver fat were 18.6 and $1.2 \%$, respectively.

\section{Body fat distribution}

Total fat and fat-free mass were determined by dual-energy X-ray absorptiometry using the QDR ${ }^{\circledR} 4500$ bone densitometer system (Hologic, Inc.).

For abdominal fat distribution, magnetic resonance (MR) abdominal images were acquired on the Philips Acheiva 3T scanner with the cardiac phase-array coil and $26.8 \mathrm{~s}$ breathhold. In brief, ten slices $(10 \mathrm{~mm}$ thick) were positioned to include the same $100 \mathrm{~mm}$ on every subject using lumbar vertebral body landmarks. Intra-abdominal fat (IAF) and subcutaneous fat (SQF) volumes were calculated using fslview version 3.1 software $^{(35)}$ combined with software developed by T. L. R. which expresses the IAF and SQF volume as $\mathrm{cm}^{3}$. Abdominal fat composition measures were performed by one trained individual who was blinded to the diet assignment. The inter- and intra-scan CV were 32.5 and $2.35 \%$ for IAF and 12.92 and $3.06 \%$ for SQF, respectively.

\section{Assays}

Insulin and glucose were measured as described in prior publications ${ }^{(36)}$. Total cholesterol and HDL-cholesterol were measured using the enzymatic colorimetric Roche Cobas c 501 assay (F. Hoffmann-La Roche). LDL-cholesterol was calculated using Friedewald's formula ${ }^{(37)}$. Oxidised LDL-cholesterol was measured using a sandwich ELISA (Mercodia AB). NEFA were quantified using the HR Series NEFA-HR kit (Wako Diagnostics Home). Highly sensitive C-reactive protein was measured by nephelometry (Siemens).

\section{Calculations and statistical analysis}

The homeostasis model of insulin resistance (HOMA-IR) was calculated as ${ }^{(38)}$ :

HOMA-IR $=$ fasting insulin $(\mu \mathrm{U}) \times$ fasting glucose $(\mathrm{mm}) / 22 \cdot 5$. 
The Matsuda index of insulin sensitivity was calculated as ${ }^{(39)}$ :

Matsuda index of insulin sensitivity

$=10000 / \sqrt{ }($ fasting glucose $\times$ fasting insulin $)$

$\times($ mean glucose OGTT $\times$ mean insulin OGTT $)$

using the 0, 60 and 120 min time points, and where OGTT is oral glucose tolerance test. All statistical analyses were performed using SPSS software (version 14.0; SPSS, Inc.). Variables that were not normally distributed are reported as medians (interquartile ranges) and were $\log _{\mathrm{e}}$ transformed to achieve a normal distribution for statistical analyses. Independent $t$ tests (for continuous variables) and $\chi^{2}$ analysis (for categorical variables) were used to compare baseline characteristics and diet composition between subjects assigned to the LSAT and HSAT diets. Paired $t$ tests were performed to determine whether there were significant changes between baseline and week 4 on each intervention diet. The general linear model for repeated measures was used to analyse for the effect of each diet intervention on liver fat while adjusting for potential confounding variables and to assess the effect of diet group assignment (LFAT $v$. HFAT) on outcome variables. There was no interaction between cognitive status (normal $v$. MCI/AD) and diet effect, thus the two cognitive status groups were combined for analysis. Linear regression models were performed to assess correlations between baseline variables and to determine what factors were associated with changes in liver fat. Baseline correlations were adjusted for age and sex. All tests were two-sided with a $P<0.05$ considered as significant.

\section{Results}

\section{Subject characteristics}

In the present study, twenty subjects were randomised to the LSAT diet (seven males/thirteen females; age 69.3 (SEm 1.6) years, BMI $26.9($ SEM 0.8$\left.) \mathrm{kg} / \mathrm{m}^{2}\right)$ and fifteen subjects were randomised to the HSAT diet (six males/nine females; age 68.6 (sem 1.8 ) years, BMI $28 \cdot 1$ (sem $0 \cdot 9) \mathrm{kg} / \mathrm{m}^{2}$ ). Subjects randomised to the LSAT and HSAT diets were well matched (Table 2).

\section{Baseline and study diet composition}

Baseline diet composition did not differ between the subjects randomised to the LSAT and HSAT diets (Table 3). The intervention diets achieved the diet composition goals of the study in terms of macronutrient composition and saturated fat content, but differed from baseline in other dietary components (Table 3). Specifically, the LSAT diet had more fibre, sugar and fructose and less cholesterol and n-3 fatty acids while the HSAT diet contained less fibre, sugar, fructose and PUFA, but more cholesterol and MUFA. In both groups, reported baseline energy intake was lower than the

Table 2. Baseline subject characteristics and response to diet interventions† (Mean values with their standard errors)

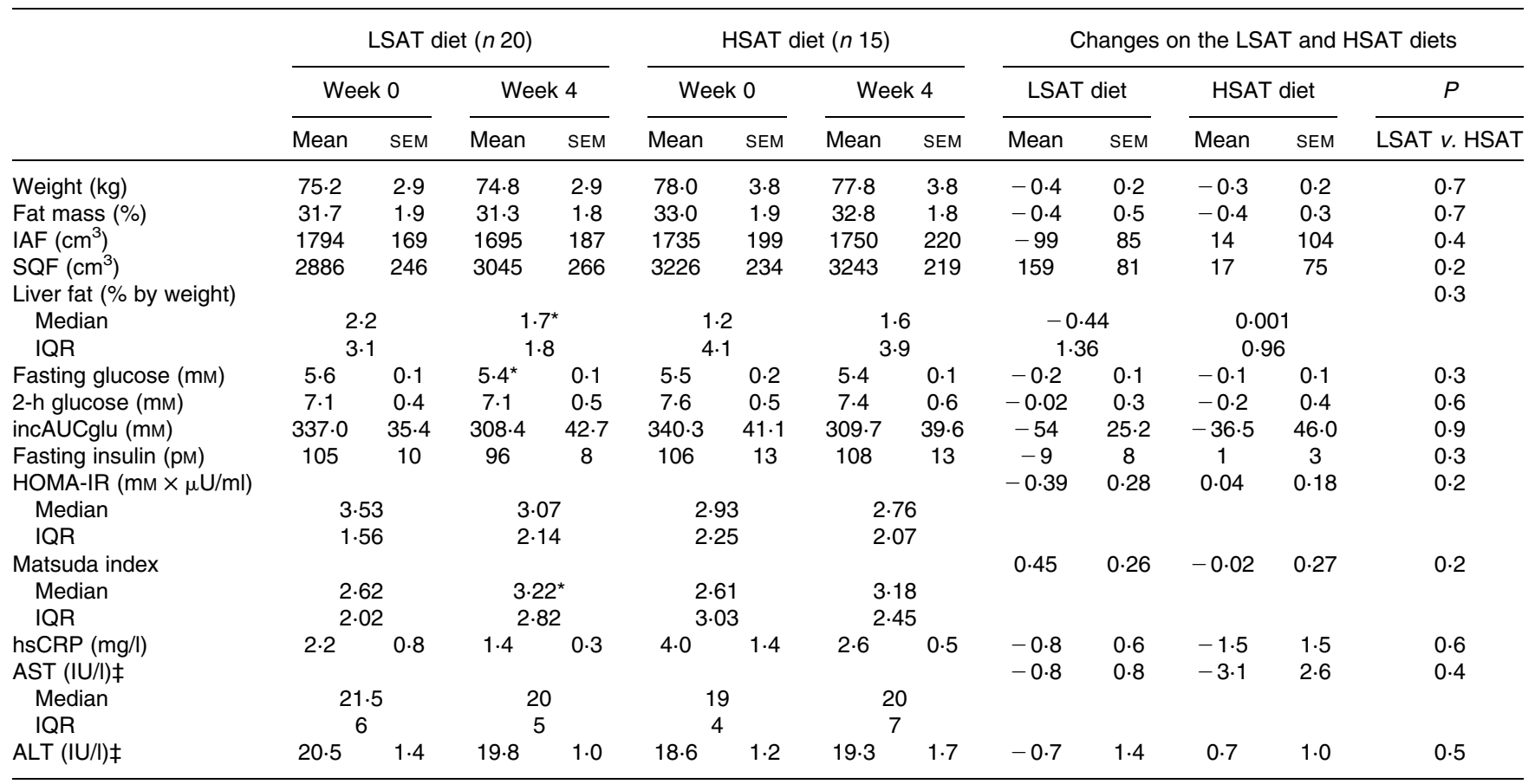

LSAT, low fat/low saturated fat/low glycaemic index diet; HSAT, high fat/high saturated fat/high glycaemic index diet; IAF, intra-abdominal fat volume; SQF, subcutaneous fat volume; IQR, interquartile range; incAUCglu, incremental area under the curve for glucose during the oral glucose tolerance test from 0 to 120 min; HOMA-IR, homeostasis model of insulin resistance; hsCRP, highly sensitive C-reactive protein; AST, aspartate transaminase; ALT, alanine aminotransaminase.

* Mean values were significantly different compared with week $0(P<0.05)$.

† All values are presented as means with their standard errors if normally distributed or medians and IQR if not normally distributed. The change in each variable was calculated for each subject then averaged per group. There were no significant differences in baseline variables between the LSAT $v$. HSAT groups.

$\ddagger$ To obtain values in nKat/l, multiply by $16 \cdot 67$. 
Table 3. Baseline and intervention diet composition

(Mean values with their standard errors)

\begin{tabular}{|c|c|c|c|c|c|c|c|c|c|c|c|c|c|}
\hline & \multicolumn{4}{|c|}{ LSAT diet $(n 20)$} & \multicolumn{4}{|c|}{ HSAT diet $(n 15)$} & \multicolumn{5}{|c|}{ Changes on the LSAT and HSAT diets } \\
\hline & \multicolumn{2}{|c|}{ Week 0† } & \multicolumn{2}{|c|}{ Week 4} & \multicolumn{2}{|c|}{ Week $0 \dagger$} & \multicolumn{2}{|c|}{ Week 4} & \multicolumn{2}{|c|}{ LSAT diet } & \multicolumn{2}{|c|}{ HSAT diet } & \multirow{2}{*}{$\frac{P}{\text { LSAT } v . \text { HSAT }}$} \\
\hline & Mean & SEM & Mean & SEM & Mean & SEM & Mean & SEM & Mean & SEM & Mean & SEM & \\
\hline Energy (kJ/d) & 8397 & 619 & $9376^{*}$ & 473 & 8351 & 343 & $9849^{*}$ & 411 & 979 & 381 & 1498 & 444 & 0.4 \\
\hline Fat (\% energy) & 34.2 & 1.5 & $23 \cdot 0^{* \star *}$ & 0.3 & 31.6 & 1.4 & $43 \cdot 0^{* \star *}$ & 0.3 & $-11 \cdot 2$ & 1.4 & 11.4 & 1.4 & $<0.001$ \\
\hline Saturated fat (\% energy) & 11.7 & 1.0 & $7 \cdot 4^{\star \star \star}$ & 0.2 & 11.1 & 0.8 & $23 \cdot 6^{\star \star \star}$ & 0.2 & -4.4 & 1.0 & $12 \cdot 6$ & 0.9 & $<0.001$ \\
\hline Carbohydrate ( $\%$ energy) & $46 \cdot 9$ & 1.5 & $57 \cdot 3^{\star \star \star}$ & 0.5 & $50 \cdot 6$ & 1.5 & $37 \cdot 9^{\star \star \star}$ & 0.5 & $10 \cdot 4$ & $6 \cdot 5$ & $-12 \cdot 6$ & 5.5 & $<0.001$ \\
\hline Protein (\% energy) & $17 \cdot 1$ & 0.7 & $17 \cdot 3$ & 0.2 & $16 \cdot 7$ & 0.9 & $16 \cdot 4$ & 0.2 & 0.2 & 0.7 & -0.2 & 0.8 & 0.72 \\
\hline Cholesterol (mg/d) & 283 & 30 & $108^{\star \star \star}$ & 6 & 223 & 27 & $354^{*}$ & 14 & -107 & 13 & 141 & 18 & $<0.001$ \\
\hline MUFA $(g / d)$ & $23 \cdot 2$ & 1.5 & $21 \cdot 2$ & 1.5 & 19.5 & 1.7 & $32 \cdot 6^{* \star *}$ & 1.5 & $-2 \cdot 0$ & 1.6 & 13.0 & $2 \cdot 1$ & $<0.001$ \\
\hline PUFA $(g / d)$ & 12.5 & 1.5 & $10 \cdot 6$ & 0.7 & $10 \cdot 8$ & $1 \cdot 2$ & $7 \cdot 7^{\star}$ & 0.3 & -1.9 & 1.4 & $-3 \cdot 1$ & 1.1 & 0.5 \\
\hline$n-3$ Fatty acids $(g / d)$ & 1.37 & 0.23 & $0.37^{\star \star \star}$ & 0.02 & $1 \cdot 14$ & 0.17 & 0.84 & 0.03 & -1.01 & 0.2 & -0.30 & 0.2 & 0.02 \\
\hline Sugar $(g / d)$ & 91 & 10 & $112^{\star}$ & 7 & 99 & 7 & $66^{\star \star \star}$ & 3 & $20 \cdot 7$ & $7 \cdot 2$ & -33.0 & $6 \cdot 5$ & $<0.001$ \\
\hline Fructose (g/d) & 9.9 & 1.4 & $13 \cdot 1^{*}$ & $1 \cdot 3$ & $14 \cdot 4$ & $2 \cdot 9$ & $8 \cdot 3^{\star}$ & $1 \cdot 0$ & $3 \cdot 3$ & 1.4 & $-6 \cdot 1$ & $2 \cdot 3$ & $<0.001$ \\
\hline Alcohol $(\mathrm{g} / \mathrm{d})$ & $5 \cdot 0$ & $2 \cdot 1$ & $2 \cdot 4$ & 1.0 & 3.8 & 1.6 & 1.6 & 0.8 & -2.6 & 1.6 & $-2 \cdot 1$ & 1.3 & 0.8 \\
\hline Fibre $(\mathrm{g} / \mathrm{d})$ & 22.9 & $2 \cdot 0$ & $40 \cdot 0^{\star \star \star}$ & 1.9 & $27 \cdot 6$ & $2 \cdot 4$ & $13 \cdot 3^{\star \star *}$ & 0.7 & $17 \cdot 2$ & $2 \cdot 0$ & $-14 \cdot 3$ & $2 \cdot 6$ & $<0.001$ \\
\hline
\end{tabular}

LSAT, low fat/low saturated fat/low glycaemic index diet; HSAT, high fat/high saturated fat/high glycaemic index diet.

Mean values were significantly different compared with baseline: ${ }^{*} P<0.05$, ${ }^{\star \star \star} P<0.001$.

† Week 0 baseline diet composition was estimated from a $3 \mathrm{~d}$ food record. There were no significant differences in baseline diet composition between the LSAT and the HSAT groups.

intervention diets. This was probably due to under-reporting as subjects remained weight stable on the intervention diets.

\section{Response to the low-fat diet intervention}

Weight, fat mass, IAF and SQF remained stable after 4 weeks on the LSAT diet (Table 2). There was a significant decrease in absolute percentage of liver fat after the LSAT diet compared with baseline $(P=0.002$; Table 2$)$, which persisted $(P<0.05)$ after adjusting for age and sex. The LSAT diet resulted in a mean percentage decrease in liver fat of $19 \cdot 8$ (SEM 6.0) \%. Similar results were obtained when data were analysed separately for the controls and MCI/AD groups (data not shown). While most subjects had low liver fat at baseline, all four subjects with baseline liver fat $>5 \%$ had decreases in liver fat after the LSAT diet. Additionally, higher liver fat at baseline was predictive of a greater absolute decrease in liver fat on the LSAT diet $(P=0.002)$.

Fasting glucose decreased significantly on the LSAT diet, but $2 \mathrm{~h}$ and incremental AUC for glucose from 0 to $120 \mathrm{~min}$ did not change (Table 2). Fasting insulin and HOMA-IR did not change significantly, but the Matsuda index improved on the LSAT diet. There were significant decreases in total cholesterol, non-HDL-, LDL- and HDL-cholesterol and a significant increase in TAG $(P<0.05$ for all; Table 4$)$. There was no significant change in NEFA or the total:HDL-cholesterol ratio, but oxidised LDL-cholesterol tended to decrease $(P=0.09$; Table 4). Liver enzymes and highly sensitive C-reactive protein were normal and did not change (Table 2).

\section{Response to the high-fat diet intervention}

Weight, fat mass, IAF and SQF remained stable after the 4-week HSAT diet (Table 2). Neither the absolute percentage of liver fat $(P=0.3$; Table 2$)$ nor the percentage change in liver fat changed significantly on the HSAT diet. There also was no effect of the HSAT diet on liver fat when analysed separately by cognitive status (data not shown).

There were no significant changes in fasting, $2 \mathrm{~h}$ and incremental AUC glucose, fasting insulin, HOMA-IR or the Matsuda index on the HSAT diet (Table 2). Liver enzymes and highly sensitive C-reactive protein did not change (Table 2). Total cholesterol, non-HDL-, LDL-, oxidised LDLand HDL-cholesterol all increased $(P<0.05)$, but TAG and NEFA did not change (Table 4).

\section{Evaluation for diet effect: comparison of the low-fat/low-saturated fat/low-glycaemic index and high-fat/high-saturated fat/high-glycaemic index diets}

There was no effect of diet group (LSAT $v$. HSAT) on liver fat $(P=0 \cdot 1)$, fasting glucose, $2 \mathrm{~h}$ glucose, incremental area under the curve glucose, fasting insulin, HOMA-IR, Matsuda index, NEFA or highly sensitive C-reactive protein. The diet assignment had a significant effect on all lipid parameters (adjusted for sex: total cholesterol $(P<0 \cdot 001)$, LDL-cholesterol $(P<0 \cdot 001)$, oxidised LDL-cholesterol $(P<0 \cdot 05)$, HDL-cholesterol $(P=0 \cdot 001)$ and TAG $(P<0 \cdot 05))$.

\section{Correlations between liver fat, insulin sensitivity, body fat distribution and lipids}

Baseline liver fat was positively associated with fasting insulin ( $r 0.53, P=0.002)$, HOMA-IR $(r 0.55, P=0.001)$, the Matsuda index $(r 0.56, P<0.001)$ and TAG $(r 0.45, P=0.009)$ and negatively associated with HDL-cholesterol $(r-0.57, P=0.001)$, but was not associated with age, sex, BMI, fat mass, IAF, $\mathrm{SQF}$, fasting or $2 \mathrm{~h}$ glucose. There were no significant correlations between the percentage change in liver fat and changes in any of the other variables on either the LSAT or the HSAT diet. 


\section{Discussion}

Beyond recommendations for weight loss, little data from well-controlled dietary studies are available regarding optimal dietary recommendations to prevent the development of or to ameliorate fatty liver. We found in an older population that 4 weeks on a weight-stable diet low in fat and saturated fat and with a LGI was associated with a small but significant decrease in liver fat. Somewhat surprisingly, the diet high in fat and saturated fat and with a HGI did not increase liver fat. Overall, these findings are important, as they suggest that diet composition is a modifiable factor that can contribute to fat accumulation in the liver.

The present finding that liver fat decreased significantly on the LSAT diet is clinically important, as it supports dietary recommendations to follow a low-fat, low-saturated fat, LGI diet. These observations in older men and women are in agreement with controlled diet studies in obese pre-menopausal women ${ }^{(27)}$ and overweight middle-aged men $^{(28)}$ in which weight remained stable. Despite the fact that absolute changes in liver fat were small and most subjects had normal liver fat at baseline, we believe that these changes are clinically relevant for several reasons. First, the present study increases our understanding of the factors involved in the accumulation of liver fat and thereby the development of NAFLD. Second, while most of our study subjects did not have liver fat in the range that would classify them as having NAFLD, all four subjects with liver fat $>5 \%$ had decreases in liver fat and baseline liver fat was a significant predictor of decreases in liver fat. Although not tested directly in this study, these findings suggest that larger and more clinically relevant decreases in liver fat would be observed in patients with NAFLD. Third, the diet intervention was for 4 weeks and dietary patterns over longer periods of time may result in larger changes with greater clinical relevance.

Determining the mechanisms whereby the LSAT diet decreased liver fat was beyond the scope of the present study. However, several observations can be made. First, changes in insulin sensitivity may not be a major mechanism whereby the LSAT diet decreased liver fat. Decreases in fasting insulin and HOMA-IR on the LSAT diet did not reach statistical significance, although the Matsuda index, which may be a better measure of muscle insulin sensitivity, showed a significant improvement. Despite a significant correlation between liver fat and insulin sensitivity measures at baseline, changes in insulin sensitivity did not correlate with changes in liver fat. Second, fasting NEFA did not change significantly with the diet intervention, suggesting that NEFA delivery at least in the basal state was not altered. However, these levels reflect the balance of release and uptake and isotope studies would be required to accurately measure rates of lipolysis and fatty acid uptake in the liver. Third, while DNL was not measured in the present study, the LGI component of the LSAT diet may have limited DNL and thereby contributed to the observed beneficial effects on liver fat. High-carbohydrate diets have been shown to increase $\mathrm{DNL}^{(40,41)}$. Furthermore, carbohydrate-restricted diets in the setting of weight loss have been shown to lead to a greater reduction in liver fat 
than energy restriction alone ${ }^{(42)}$. However, carbohydrateinduced increases in DNL were attenuated by diets containing starch instead of short-chain glucose polymers or sugar ${ }^{(41)}$. Currently, there are no interventional data in humans examining the impact of the GI on liver fat accumulation. However, data in rodents suggest that a HGI may be important in modulating lipogenesis ${ }^{(43)}$, body fat distribution and lipid oxidation $^{(44)}$. Of note, neither intervention diet in the present study significantly altered abdominal fat distribution.

Other potential mechanisms to explain the improvement in liver fat on the LSAT diet include decreased delivery of chylomicrons due to a reduction in dietary fat content and/or changes in lipid oxidation. Other dietary factors besides fat and saturated fat content and the GI may have contributed to beneficial effects, including higher fibre content and dietary cholesterol content that was approximately $50 \%$ lower than baseline. Animal studies have shown that dietary cholesterol can contribute to the development of $\mathrm{NASH}^{(45)}$. However, human studies have failed to show an effect of high $v$. low dietary cholesterol intake on liver fat or liver enzymes ${ }^{(19)}$, and we did not observe changes in liver enzymes on either diet. Elucidation of exact mechanisms and specific beneficial dietary components requires further study.

The lack of an effect of the HSAT diet on liver fat was surprising and is in contrast to the findings by Westerbacka ${ }^{(27)}$ and van Herpen ${ }^{(28)}$ who observed increases in liver fat on a high-fat diet relative to a low-fat diet after 2 and 3 weeks, respectively. There are multiple possible explanations for this discrepancy. First, age may be an important factor. The Westerbacka study enrolled pre-menopausal women and the study by van Herpen middle-aged men. Older individuals may be more resistant to the effects of a HSAT diet to increase liver fat. Second, while the total fat content of the diet that we used was high, it was not as high as those used by Westerbacka $^{(27)}$ and van Herpen ${ }^{(28)}$ who employed diets containing extreme levels of fat (56 and 55\% energy from fat). Thus, a higher total fat content may be required to observe increases in liver fat. However, the relevance of such extreme high-fat diets to the pathophysiology of NAFLD is debatable. The baseline diet composition of our subjects contained 34\% energy from fat which is close to the estimates for diet macronutrient composition in the $\mathrm{USA}^{(46)}$. The converse is also possible; that is, baseline dietary fat content is already high and a ceiling effect prevents further increases in liver fat. Third, the subject population that we studied was on average mildly overweight rather than obese and had normal liver fat at baseline. Ectopic fat deposition may be enhanced in subjects who are more insulin-resistant or obese. However, overfeeding studies that have demonstrated significant increases in liver fat were performed in young, lean subjects ${ }^{(19-21,47)}$ with low levels of liver fat, suggesting that diet composition is probably less important than the overall energy balance in liver fat accumulation. Finally, changes in other fat components may have had beneficial effects. MUFA were increased by $67 \%$, PUFA were decreased by $29 \%$ and total cholesterol was increased by $59 \%$ on the HSAT diet. While long-chain PUFA have been shown to inhibit lipogenesis and promote lipid oxidation ${ }^{(48)}$, the amount of $n-3$ fatty acids in the present study did not change significantly on the HSAT diet. MUFA, the predominant fatty acid in olive oil, has shown beneficial effects on liver fat in animal models ${ }^{(49)}$, and therefore could have mitigated the negative effects of the high fat and saturated fat content. Intervention studies to evaluate the effect of increased dietary MUFA on liver fat in humans have not been performed. High cholesterol content, as noted above, has not been shown to influence liver fat accumulation in humans ${ }^{(19)}$.

While the present data favour the use of a diet low in fat, and therefore high in carbohydrates, others have shown that high-carbohydrate diets $(>60 \%)$ increased TAG and decreased HDL-cholesterol ${ }^{(50)}$ and low-carbohydrate, nonketogenic diets decreased glucose levels in individuals with diabetes $^{(51)}$. We also observed potentially adverse lipid changes on the LSAT diet with increased TAG and decreased HDL-cholesterol. On the other hand, the LSAT diet resulted in a beneficial and robust decrease in non-HDL-cholesterol, while non-HDL-cholesterol increased on the HSAT diet. The combined significance of these beneficial and potentially adverse lipid effects on CVD is unknown. While highcarbohydrate diets in diabetes may increase glucose levels, especially if they contain HGI foods, LGI diets have actually been shown to improve glucose and HbA1c in subjects with type 2 diabetes ${ }^{(52,53)}$. Consistent with this, the LSAT diet which contained LGI foods resulted in small but significant decreases in fasting glucose, suggesting a benefit, at least in non-diabetic individuals.

The strengths of the study include the controlled nature of the diet intervention, the unique population of older adults studied, and the achievement of weight stability to be able to determine the effect of macronutrient composition on liver fat independent of changes in energy balance. There are several limitations to the present study. First, while the LSAT diet resulted in a significant improvement in the Matsuda index, we did not observe the expected decrease in insulin sensitivity on the HSAT diet. Fasting measures, which are more reflective of hepatic insulin sensitivity, did not change on either diet. It is likely that the lack of a significant change in HOMA-IR was due to small sample size. However, we cannot rule out the possibility that changes in specific dietary components may have had important effects on muscle insulin sensitivity without altering hepatic insulin sensitivity. While some studies have shown that diets high in saturated fat result in decreased insulin sensitivity ${ }^{(4-6)}$, and that substitution of carbohydrates and monounsaturated fats can improve insulin sensitivity ${ }^{(54)}$, others have failed to see changes in insulin sensitivity after $15 \mathrm{~d}$ of a high-fat (50\%) $\operatorname{diet}^{(55)}$. Thus, firm conclusions regarding the interplay between diet-induced changes in liver fat and insulin sensitivity cannot be drawn. Second, the study specifically targeted older individuals and the findings may not apply to younger populations. Third, cognitive status could be a potential confounder as we combined the subjects with and without $\mathrm{MCI} / \mathrm{AD}$ together for the present analyses. Individuals with $\mathrm{MCI} / \mathrm{AD}$ showed differential responses to the diets in cerebral spinal fluid insulin and inflammatory markers ${ }^{(29)}$. However, we did not see an interaction between cognitive status and changes in liver fat, and the observed changes in liver fat on 
the diets were consistent with the present overall results when analysed separately by cognitive status.

In summary, we have shown that diet composition in the absence of changes in weight may play a role in the accumulation of liver fat in older individuals. Specifically, a diet low in fat, saturated fat and with a LGI resulted in small decreases in liver fat. The negative results on the HSAT diet suggest that the common assumption that high fat and saturated fat content above and beyond the standard diet contributes to NAFLD may be incorrect. In light of previous studies on the effects of weight loss and overfeeding on liver fat, the small changes on the LSAT diet coupled with the lack of change on the HSAT diet suggest that diet composition is probably less important than energy balance and that clinical advice for the prevention of NAFLD should be focused more on the prevention of weight gain or the promotion of weight loss. However, the small decreases in liver fat on the LSAT diet may be clinically relevant in subjects with high liver fat and if changes in dietary intake can be sustained for a longer period of time. Thus, while one must be cautious in making recommendations about appropriate dietary recommendations based on such a small study, the present results add support for recommendations to follow a diet low in fat, saturated fat and with a LGI for the prevention of NAFLD.

\section{Acknowledgements}

We are grateful to the study participants for their contribution and time. This study was supported in part by the VA Office of Research and Development Medical Research Service, grant no. R37 AG-10880, UL1RR025014, P30 DK017047 and the University of Washington Diagnostic Imaging Science Center. The funding agencies had no role in the design, implementation, analysis or interpretation of the data. The authors' responsibilities were as follows: K. M. U. designed the MRS sub-study, analysed the data and wrote the manuscript; J. L. B.-C. designed the diet interventions; M. D. A. and J. M. T. coordinated the study and data entry; T. L. R. designed the MRS protocol and interpreted the scans; S. C. designed the parent study and assisted with the data analysis. All authors read and approved the final manuscript. None of the authors had a conflict of interest to disclose.

\section{References}

1. Browning JD, Szczepaniak LS, Dobbins R, et al. (2004) Prevalence of hepatic steatosis in an urban population in the United States: impact of ethnicity. Hepatology $\mathbf{4 0}$, 1387-1395.

2. Frith J, Day CP, Henderson E, et al. (2009) Non-alcoholic fatty liver disease in older people. Gerontology 55, 607-613.

3. Donnelly KL, Smith CI, Schwarzenberg SJ, et al. (2005) Sources of fatty acids stored in liver and secreted via lipoproteins in patients with nonalcoholic fatty liver disease. J Clin Invest 115, 1343-1351.

4. Lovejoy JC, Smith SR, Champagne CM, et al. (2002) Effects of diets enriched in saturated (palmitic), monounsaturated (oleic), or trans (elaidic) fatty acids on insulin sensitivity and substrate oxidation in healthy adults. Diabetes Care 25, 1283-1288.

5. Vessby B, Unsitupa M, Hermansen K, et al. (2001) Substituting dietary saturated for monounsaturated fat impairs insulin sensitivity in healthy men and women: The KANWU Study. Diabetologia 44, 312-319.

6. Xiao C, Giacca A, Carpentier A, et al. (2006) Differential effects of monounsaturated, polyunsaturated and saturated fat ingestion on glucose-stimulated insulin secretion, sensitivity and clearance in overweight and obese, non-diabetic humans. Diabetologia 49, 1371-1379.

7. Proietto J, Filippis A, Nakhla C, et al. (1999) Nutrient-induced insulin resistance. Mol Cell Endocrinol 151, 143-149.

8. Bessesen DH (2001) The role of carbohydrates in insulin resistance. J Nutr 131, 2782S-2786S.

9. McLaughlin T, Yee G, Glassford A, et al. (2011) Use of a twostage insulin infusion study to assess the relationship between insulin suppression of lipolysis and insulinmediated glucose uptake in overweight/obese, nondiabetic women. Metabolism 60, 1741-1747.

10. Browning JD \& Horton JD (2004) Molecular mediators of hepatic steatosis and liver injury. J Clin Invest 114, 147-152.

11. Wolever TM, Yang M, Zeng XY, et al. (2006) Food glycemic index, as given in glycemic index tables, is a significant determinant of glycemic responses elicited by composite breakfast meals. Am J Clin Nutr 83, 1306-1312.

12. Fried SK \& Rao SP (2003) Sugars, hypertriglyceridemia, and cardiovascular disease. Am J Clin Nutr 78, 873S-880S.

13. Brynes AE, Mark Edwards C, Ghatei MA, et al. (2003) A randomised four-intervention crossover study investigating the effect of carbohydrates on daytime profiles of insulin, glucose, non-esterified fatty acids and triacylglycerols in middle-aged men. Br J Nutr 89, 207-218.

14. Tiikkainen M, Bergholm R, Vehkavaara S, et al. (2003) Effects of identical weight loss on body composition and features of insulin resistance in obese women with high and low liver fat content. Diabetes 52, 701-707.

15. Vilar L, Oliveira CP, Faintuch J, et al. (2008) High-fat diet: a trigger of non-alcoholic steatohepatitis? Preliminary findings in obese subjects. Nutrition 24, 1097-1102.

16. Toshimitsu K, Matsuura B, Ohkubo I, et al. (2007) Dietary habits and nutrient intake in non-alcoholic steatohepatitis. Nutrition 23, 46-52.

17. Assy N, Nasser G, Kamayse I, et al. (2008) Soft drink consumption linked with fatty liver in the absence of traditional risk factors. Can J Gastroenterol 22, 811-816.

18. Valtuena S, Pellegrini N, Ardigo D, et al. (2006) Dietary glycemic index and liver steatosis. Am J Clin Nutr 84, 136-142, quiz 268-139.

19. Bortolotti M, Kreis R, Debard C, et al. (2009) High protein intake reduces intrahepatocellular lipid deposition in humans. Am J Clin Nutr 90, 1002-1010.

20. Sobrecases H, Le KA, Bortolotti M, et al. (2010) Effects of short-term overfeeding with fructose, fat and fructose plus fat on plasma and hepatic lipids in healthy men. Diabetes Metab 36, 244-246.

21. Ngo Sock ET, Le KA, Ith M, et al. (2010) Effects of a shortterm overfeeding with fructose or glucose in healthy young males. Br J Nutr 103, 939-943.

22. Zivkovic AM, German JB \& Sanyal AJ (2007) Comparative review of diets for the metabolic syndrome: implications for nonalcoholic fatty liver disease. Am J Clin Nutr 86, 285-300.

23. Huang MA, Greenson JK, Chao C, et al. (2005) One-year intense nutritional counseling results in histological 
improvement in patients with non-alcoholic steatohepatitis: a pilot study. Am J Gastroenterol 100, 1072-1081.

24. Promrat K, Kleiner DE, Niemeier HM, et al. (2010) Randomized controlled trial testing the effects of weight loss on nonalcoholic steatohepatitis. Hepatology 51, 121-129.

25. Lazo M, Solga SF, Horska A, et al. (2010) Effect of a 12-month intensive lifestyle intervention on hepatic steatosis in adults with type 2 diabetes. Diabetes Care 33, 2156-2163.

26. Shah K, Stufflebam A, Hilton TN, et al. (2009) Diet and exercise interventions reduce intrahepatic fat content and improve insulin sensitivity in obese older adults. Obesity 17, 2162-2168.

27. Westerbacka J, Lammi K, Hakkinen AM, et al. (2005) Dietary fat content modifies liver fat in overweight nondiabetic subjects. J Clin Endocrinol Metab 90, 2804-2809.

28. van Herpen NA, Schrauwen-Hinderling V, Schaart G, et al. (2011) Three weeks on a high-fat diet increases intrahepatic lipid accumulation and decreases metabolic flexibility in healthy overweight men. J Clin Endocrinol Metab 96, E691-E695.

29. Bayer-Carter JL, Green PS, Montine TJ, et al. (2011) Diet intervention and cerebrospinal fluid biomarkers in amnestic mild cognitive impairment. Arch Neurol 68, 743-752.

30. USDA Standard Reference 18 and Food and Nutrient Database for Dietary Studies 1.0. http://www.ars.usda.gov/ Services/docs.htm?docid $=12082$

31. Mifflin MD, St Jeor ST, Hill LA, et al. (1990) A new predictive equation for resting energy expenditure in healthy individuals. Am J Clin Nutr 51, 241-247.

32. Harris JA \& Benedict FG (1919) A Biometric Study of Basal Metabolism in Man. Washington, DC: Carnegie Institution of Washington.

33. Longo R, Ricci C, Masutti F, et al. (1993) Fatty infiltration of the liver. Quantification by ${ }^{1} \mathrm{H}$ localized magnetic resonance spectroscopy and comparison with computed tomography. Invest Radiol 28, 297-302.

34. Szczepaniak LS, Nurenberg P, Leonard D, et al. (2005) Magnetic resonance spectroscopy to measure hepatic triglyceride content: prevalence of hepatic steatosis in the general population. Am J Physiol 288, E462-E468.

35. FSLview. http://www.fmrib.ox.ac.uk/fsl/fslview/index.html

36. Craft S, Newcomer J, Kanne S, et al. (1996) Memory improvement following induced hyperinsulinemia in Alzheimer's disease. Neurobiol Aging 17, 123-130.

37. Friedewald WT, Levy RI \& Fredrickson DS (1972) Estimation of the concentration of low-density lipoprotein cholesterol in plasma, without use of the preparative ultracentrifuge. Clin Chem 18, 499-502.

38. Matthews DR, Hosker JP, Rudenski AS, et al. (1985) Homeostasis model assessment: insulin resistance and $\beta$-cell function from fasting plasma glucose and insulin concentrations in man. Diabetologia 28, 412-419.

39. Matsuda M \& DeFronzo RA (1999) Insulin sensitivity indices obtained from oral glucose tolerance testing: comparison with the euglycemic insulin clamp. Diabetes Care 22, $1462-1470$.
40. Hudgins LC, Hellerstein M, Seidman C, et al. (1996) Human fatty acid synthesis is stimulated by a eucaloric low fat, high carbohydrate diet. J Clin Invest 97, 2081-2091.

41. Hudgins LC, Seidman CE, Diakun J, et al. (1998) Human fatty acid synthesis is reduced after the substitution of dietary starch for sugar. Am J Clin Nutr 67, 631-639.

42. Browning JD, Baker JA, Rogers T, et al. (2011) Short-term weight loss and hepatic triglyceride reduction: evidence of a metabolic advantage with dietary carbohydrate restriction. Am J Clin Nutr 93, 1048-1052.

43. Kabir M, Rizkalla SW, Quignard-Boulange A, et al. (1998) A high glycemic index starch diet affects lipid storage-related enzymes in normal and to a lesser extent in diabetic rats. J Nutr 128, 1878-1883.

44. Isken F, Klaus S, Petzke KJ, et al. (2009) Impairment of fat oxidation under high vs low glycemic index diet occurs prior to the development of an obese phenotype. Am J Physiol Endocrinol Metab 298, E287-E295.

45. Subramanian S, Goodspeed L, Wang S, et al. (2011) Dietary cholesterol exacerbates hepatic steatosis and inflammation in obese LDL receptor-deficient mice. J Lipid Res $\mathbf{5 2}$, 1626-1635.

46. USDA, What we eat in America. http://www.ars.usda.gov/ Services/docs.htm?docid=18349

47. Kechagias S, Ernersson A, Dahlqvist O, et al. (2008) Fastfood-based hyper-alimentation can induce rapid and profound elevation of serum alanine aminotransferase in healthy subjects. Gut 57, 649-654.

48. Jump DB \& Clarke SD (1999) Regulation of gene expression by dietary fat. Annu Rev Nutr 19, 63-90.

49. Hussein O, Grosovski M, Lasri E, et al. (2007) Monounsaturated fat decreases hepatic lipid content in non-alcoholic fatty liver disease in rats. World J Gastroenterol 13, 361-368.

50. Retzlaff BM, Walden CE, Dowdy AA, et al. (1995) Changes in plasma triacylglycerol concentrations among free-living hyperlipidemic men adopting different carbohydrate intakes over 2 y: the Dietary Alternatives Study. Am J Clin Nutr 62, 988-995.

51. Gannon MC \& Nuttall FQ (2004) Effect of a high-protein, low-carbohydrate diet on blood glucose control in people with type 2 diabetes. Diabetes 53, 2375-2382.

52. Livesey G, Taylor R, Hulshof $\mathrm{T}$, et al. (2008) Glycemic response and health - a systematic review and meta-analysis: relations between dietary glycemic properties and health outcomes. Am J Clin Nutr 87, 258S-268S.

53. Thomas DE \& Elliott EJ (2010) The use of low-glycaemic index diets in diabetes control. Br J Nutr 104, 797-802.

54. Perez-Jimenez F, Lopez-Miranda J, Pinillos MD, et al. (2001) A Mediterranean and a high-carbohydrate diet improve glucose metabolism in healthy young persons. Diabetologia $\mathbf{4 4}$, 2038-2043.

55. Eckel RH, Hernandez TL, Bell ML, et al. (2006) Carbohydrate balance predicts weight and fat gain in adults. Am J Clin Nutr 83, 803-808. 